\title{
Redução de cor ICUMSA em soluções concentradas de açúcar bruto por processo oxidativo com peróxido de hidrogênio
}

\section{ICUMSA colour reduction in concentrated raw sugar solutions by an oxidative process with hydrogen peroxide}

\section{Juliana Lorenz Mandro', Nathália Torres Correa Magri ${ }^{1}$, Juliana Aparecida de Souza Sartori', Claudio Lima de Aguiar ${ }^{1 *}$}

1 Universidade de São Paulo (USP), Escola Superior de Agricultura "Luiz de Queiroz", Departamento de Agroindústria, Alimentos e Nutrição, Laboratório Hugot de Tecnologia em Sucroderivados, Piracicaba/SP - Brazil

\section{*Corresponding Author}

Claudio Lima de Aguiar, Universidade de São Paulo (USP), Escola Superior de Agricultura "Luiz de Queiroz", Departamento de Agroindústria, Alimentos e Nutrição, Av. Padua Dias, 11, Caixa Postal: 9, CEP: 13418-900, Piracicaba/SP - Brazil, e-mail: claguiar@usp.br

Cite as: ICUMSA colour reduction in concentrated raw sugar solutions by an oxidative process with hydrogen peroxide. Braz. J. Food Technol., v. 20, e2016114, 2017

Received: Aug. 29, 2016; Accepted: Apr. 03, 2017

\section{Resumo}

A clarificação/purificação do açúcar refinado a partir de açúcares brutos, como Very High Polarization (VHP), é realizada atualmente pelo uso de resinas de troca-iônica ou carvão ativado. No entanto, são metodologias de alto custo, que necessitam de constante regeneração/recuperação e apresentam perda de capacidade em função do tempo de processamento. Neste sentido, estudos sobre tecnologias alternativas ao uso de resinas de troca-iônica e carvão ativado vêm sendo cada vez mais relacionados, para aumentar a economia do setor. O peróxido de hidrogênio em solução comercial $(35 \%, v / v)$ pode também ser entendido como agente promotor de redução de cor ICUMSA, na cadeia produtiva do açúcar refinado. Com isso, foram realizados tratamentos a fim de testar a eficiência do agente oxidante na clarificação de açúcar bruto do tipo VHP. Os tratamentos foram avaliados frente a diferentes doses de peróxido de hidrogênio, $\mathrm{pH}$ e temperaturas, durante um tempo reacional de uma hora. Todas as amostras tratadas apresentaram redução de cor ICUMSA, sendo que o maior decréscimo esteve na faixa de 67 a $70 \%$. O aumento da dose de agente oxidante foi proporcional ao aumento de precipitados no meio, em que o tratamento com maior concentração de peróxido de hidrogênio apresentou índice de turbidez de 142 NTU em relação ao valor da amostra bruta (17 NTU), sendo o ensaio com maior turbidez. A degradação de sacarose ocorreu de forma mais pronunciada nos tratamentos com valores de $\mathrm{pH}$ abaixo de 5,0. Com isso, o peróxido de hidrogênio se mostrou uma alternativa viável para redução de cor ICUMSA e precipitação de não-açúcares e os tratamentos com melhor redução de cor também não foram críticos na degradação de sacarose.

Palavras-chave: Clarificação; Oxidação; Sacarose.

\section{Abstract}

Currently, the clarification/purification of refined sugar as from raw sugars such as very high polarization (VHP) is carried out using ion exchange resins or activated carbon. However, these are costly methodologies, which require constant regeneration/recovery and exhibit a loss of their ability as a function of processing time. Hence studies on alternative technologies to the use of ion-exchange resins and activated carbon are being increasingly reported to increase the economy of the sector. A commercial solution ( $35 \% \mathrm{v} / \mathrm{v}$ ) of hydrogen peroxide can also be understood as an ICUMSA colour reducing promoter in the refined sugar production chain. Thus treatments were carried out to test the efficiency of the oxidizing agent in the clarification of type VHP raw sugar. The treatments were evaluated using different doses of hydrogen peroxide, and different $\mathrm{pH}$ values and temperatures for a reaction time of one hour. All the treated samples showed a reduction in the ICUMSA colour, the greatest decrease being in the range from 67 to $70 \%$. The increase in dose of oxidizing agent was proportional to the increase in precipitates in the medium, the treatment with the highest hydrogen peroxide concentration showing turbidity of 142 NTU as compared to the value of the crude sample of 17 NTU, and was the assay with the greatest 
turbidity. Sucrose degradation was more pronounced in treatments below pH 5.0. Thus hydrogen peroxide was shown to be a viable alternative to reduce ICUMSA colour and precipitate non-sugars and the treatments with better colour reduction were also not critical with respect to sucrose degradation.

Keywords: Clarification; Oxidation; Sucrose.

\section{Introdução}

Para a indústria sucroenergética, o conhecimento da matéria-prima e a compreensão das suas propriedades químicas e reacionais, durante e após processamento, são de suma importância. Na medida em que o açúcar representa uma das maiores commodities brasileiras, faz-se indispensável a busca por novas tecnologias, objetivando maiores rendimentos e economia no setor. Atualmente, nas refinarias, a clarificação/purificação do açúcar refinado é realizada por resinas de troca-iônica ou carvão ativado granulado. Para Crema (2012), o processo consiste na dissolução do açúcar cristal com alta polarização em água a $75^{\circ} \mathrm{C}$, gerando-se uma calda. Esta solução é submetida a um processo de purificação, pelo qual a calda é submetida ao processo de aeração e filtração, tornando-se uma calda clara e brilhante, mas ainda com grande parte da cor do açúcar cristal. Esta cor restante se suprime pela ação de meio descolorante, como carvão granulado e resinas iônicas.

A calda gerada no processo de purificação/clarificação é aquecida a $85^{\circ} \mathrm{C}$ e transferida para batedeiras, nas quais se torna uma massa quente e úmida de açúcar. Nesta etapa, os cristais não têm uma forma definida. Para secar e esfriar, o açúcar é enviado para secadores com passagem de ar quente e frio. O açúcar, então, é peneirado para separar os aglomerados e obter-se a uniformidade dos cristais. A partir da fração de finos, é obtido o açúcar destinado à produção de açúcar de confeiteiro e da fração de grossos, obtém-se o açúcar refinado (MACHADO, 2012).

No entanto, a produção de açúcar refinado por meio de resinas de troca-iônica tem alto custo agregado, uma vez que o material necessita de regeneração constante e uma fração da capacidade da resina é perdida progressivamente, devido ao tempo de uso (RODRIGUES, 1998). Além disso, para Marquez (2007), com o uso de resinas de troca-iônica, há casos em que a sacarose pode ser altamente hidrolisada, formando um meio com grande incidência de açúcar invertido e, assim, dificultando o processo de cristalização do açúcar. Com o carvão ativado, o cenário é semelhante, pois a fabricação desse adsorvente, por vezes, é de alto custo, devido à origem e ao valor da matéria-prima (BACCAR et al., 2009). Aliado a isso, ocorrem também perdas durante o processo de recuperação do adsorvente, tornando sua utilização, muitas vezes, onerosa. Neste cenário, métodos alternativos como o uso de peróxido de hidrogênio $\left(\mathrm{H}_{2} \mathrm{O}_{2}\right)$, aplicados em calda de açúcar bruto, vêm sendo estudados para aumentar a economia do setor, uma vez que tal método é considerado de baixo custo e não exige grandes modificações na infraestrutura industrial das refinarias (SARTORI et al., 2015b).

O peróxido de hidrogênio atua nos compostos coloridos, como melanoidinas, melaninas, caramelos, amido, aminoácidos e polifenóis, os quais são formados, em sua grande maioria, durante o processo de obtenção do açúcar, refletindo na tonalidade do produto final. Seu mecanismo de ação, quando catalisado por temperatura e/ou pH, consiste na formação de radical $\bullet \mathrm{HO}$, agente altamente oxidante e reativo, que, juntamente com uma variedade de classes de compostos, promove a decomposição dos pigmentos orgânicos (BOURZUTSCHKY, 2005). Para Djogic et al. (2005), o peróxido de hidrogênio também tem a capacidade de agir na presença de compostos inorgânicos, catalisando-os e formando precipitados no meio.

As condições de reação requeridas para o uso do $\mathrm{H}_{2} \mathrm{O}_{2}$ são simples e próximas às condições ambientais, ou seja, 0,1 MPa a 0,5 MPa de pressão e temperaturas menores que $80{ }^{\circ} \mathrm{C}$, permitindo a decomposição de uma série de compostos orgânicos, com baixo consumo energético. Assim, propõe-se avaliar o uso do peróxido de hidrogênio em solução comercial (35\%, v/v) como reagente alternativo para obtenção de açúcar refinado com baixa cor ICUMSA, a fim de melhorar as condições de tratamento, bem como avaliar a degradação da sacarose e o poder precipitante do peróxido de hidrogênio.

\section{Material e métodos}

\subsection{Açúcar bruto}

Para a realização do trabalho, foi adquirido açúcar do tipo VHP (very high polarization) de usina da região de Piracicaba-SP, com características de pH $(6,56)$, umidade $(0,026 \%)$, polarização $\left(97,21^{\circ} \mathrm{Z}\right)$, cinzas $(0,66 \%)$, granulometria $(19,09 \%$ CV e AM 0,9 mm) e Cor ICUMSA $(417,58 \mathrm{UI})$.

\subsection{Condução dos tratamentos de clarificação da calda a $66^{\circ}$ Brix de açúcar bruto}

Foi realizado um delineamento composto central rotacional $2^{3}$ completo com três pontos centrais e seis pontos axiais (Tabela 1), com o intuito de obter as melhores condições para redução de cor ICUMSA e menor degradação da sacarose. Para isso, o açúcar foi misturado à água destilada até atingir uma concentração de $66^{\circ}$ Brix (teor de sólidos solúveis), visando obter uma calda, com as 
Redução de cor ICUMSA em soluções concentradas de açúcar bruto por processo oxidativo com peróxido de hidrogênio Mandro, J. L. et al.

Tabela 1. Delineamento composto central rotacional (DCCR).

\begin{tabular}{lccccc}
\multicolumn{1}{c}{ Parâmetros } & $-\alpha(\mathbf{- 1 , 6 8 )}$ & $\mathbf{- 1}$ & $\mathbf{0}$ & $\mathbf{+ 1}$ & $\mathbf{+}(\mathbf{+ 1 , 6 8 )}$ \\
$\mathbf{p H}$ & 1,64 & 3,0 & 5,0 & 7,0 & 8,36 \\
Temperatura $\left({ }^{\circ} \mathbf{C}\right)$ & 16,4 & 30 & 50 & 70 & 83,6 \\
$\mathbf{H}_{\mathbf{2}} \mathbf{O}_{\mathbf{2}}$ (ppm) & 0 & 202,4 & 500 & 797,6 & 1000 \\
\hline
\end{tabular}

mesmas condições de uma refinaria. A diluição foi ajustada em refratômetro de bancada (Bellingham + Stanley Ltda.).

Foram obtidos e realizados 17 tratamentos com distintas combinações, sendo que, em cada tratamento, adicionaram-se, em Erlenmeyer, $200 \mathrm{~mL}$ de calda de açúcar bruto a $66^{\circ}$ Brix e o peróxido de hidrogênio (grau comercial, em concentração de 35\%), nas concentrações estabelecidas pelo planejamento. $\mathrm{O}$ pH para cada ensaio foi corrigido com ácido clorídrico $0,1 \mathrm{~N}$ ou hidróxido de sódio $0,1 \mathrm{~N}$, e foi determinado utilizando-se um pHmetro (Tecnal, Tec 2). A temperatura da calda foi ajustada no banho shaker e acompanhada com termômetro digital (Incoterm). A clarificação foi conduzida em shaker termostatizado com agitação orbital constante a 100 rpm por uma hora. Ao final, foi adicionada catalase (Sigma-Aldrich, liofilizada em pó, com 2.000 a 5.000 unidades/mg de proteína), proveniente de fígado de boi, para promover o término da reação.

\subsection{Análise de cor ICUMSA}

Para analisar o índice de cor ICUMSA (Método G.S. 1/3-7) (ICUMSA, 2011), as amostras foram medidas quanto ao 'Brix, em refratômetro de bancada (Bellingham + Stanley Ltda.) e diluídas em triplicata a $30^{\circ}$ Brix. Posteriormente, foram filtradas sob condição de vácuo em membrana de filtro PTFE de 0,45 um diâmetro de poro (Millipore Co., São Paulo, Brasil). O pH das amostras foi corrigido para

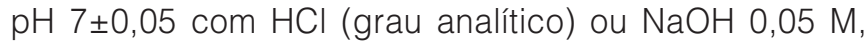
sendo, em seguida, lidas em espectrofotômetro UV Mini 1240 (Shimadzu Co., Kyoto, Japão) a uma absorbância de $420 \mathrm{~nm}$, com cubeta de quartzo de $1 \mathrm{~cm}$. O índice de cor ICUMSA das amostras de calda de açúcar foi calculado utilizando-se a Equação 1:

Cor ICUMSA $(420 \mathrm{~nm})=\frac{\mathrm{ABS}}{(\mathrm{b} \mathrm{c})} \times 1000$

Em que:

ABS: Absorbância a $420 \mathrm{~nm}$;

b: Percurso ótico da célula $(\mathrm{cm})$;

c: Concentração de sacarose $(\mathrm{g} / \mathrm{mL})$ na solução açucarada em função do ${ }^{\circ}$ Brix a $20{ }^{\circ} \mathrm{C}$.

\subsection{Determinação de turbidez}

O aumento da turbidez foi avaliado em turbidímetro TB-10000 (Tecnopom, Piracicaba, Brasil), de acordo com Norma Brasileira Regulamentadora 9757 (ABNT,
1987), em que as amostras foram diluídas a $30{ }^{\circ}$ Brix devido à sua viscosidade, sendo, posteriormente, lidas em turbidímetro e os valores expressos em unidades de turbidez nefelométricas (NTU).

\subsection{Análise de sacarose por cromatografia líquida de alta eficiência com detector de espalhamento de Iuz evaporativo}

A sacarose foi determinada usando-se cromatógrafo líquido de alta eficiência com detector de espalhamento de luz evaporativo (UFLC/ELSD-LT). Como fase móvel, foi utilizada uma solução mista de acetronitrila e água, na proporção de 85:15, previamente filtrada em membranas de 0,45 $\mu \mathrm{m}$, bem como as amostras. A coluna usada foi uma Kromasil $100 \mathrm{NH}_{2} 5 \mu \mathrm{m} 250 \times 46 \mathrm{~mm}$, termostatizada a $30{ }^{\circ} \mathrm{C}$, no sentido de evitar flutuações na resposta do detector. As condições de trabalho foram: fluxo de $1,0 \mathrm{~mL} / \mathrm{min}$, temperatura do detector igual a $35^{\circ} \mathrm{C}$, pressão de 350 kPa e nitrogênio como gás de nebulização. As amostras (volume de injeção de $5 \mu \mathrm{L}$ ) foram analisadas em triplicata. Antes das análises quantitativas de sacarose, soluções-padrão foram preparadas para a elaboração das curvas de calibração (0,1 a 0,5 g/L) (SILVA et al., 2015).

\subsection{Análise estatística dos dados}

Os resultados obtidos foram analisados por meio de superfícies de resposta, relacionando-as com os fatores estudados, dois a dois, e por meio do Quadro de ANOVA, utilizando o programa Statistica v. 15.

\section{Resultados e discussão}

A turbidez de todos os tratamentos aumentou em relação ao da amostra bruta (Tratamento 0), como mostra a Tabela 2, sugerindo que houve a formação de partículas maiores na calda quando em reação com o agente clarificante $\left(\mathrm{H}_{2} \mathrm{O}_{2}\right)$. Observou-se, ainda, que a amostra do Tratamento 16 (sem adição de $\mathrm{H}_{2} \mathrm{O}_{2}$ ) apresentou valor de turbidez de 29 NTU, obtendo o menor valor dentre os 17 tratamentos relacionados. Para o Tratamento 17, que continha a maior concentração de $\mathrm{H}_{2} \mathrm{O}_{2}$, o aumento foi de 142 NTU em relação ao valor da amostra bruta de 17 NTU, o que reforça a ideia de que $\mathrm{H}_{2} \mathrm{O}_{2}$ agiu como precipitante na solução. Para Chunhong (1993 apud SARTORI et al., 2015a), a formação de precipitados acontece a partir da reação entre derivados de não-açúcares com o $\mathrm{H}_{2} \mathrm{O}_{2}$. Para Cahill e Burkhart (1990) e Nikonov et al. (2010), diversos tipos de compostos são altamente hidrolisáveis 
Redução de cor ICUMSA em soluções concentradas de açúcar bruto por processo oxidativo com peróxido de hidrogênio Mandro, J. L. et al.

na presença de $\mathrm{H}_{2} \mathrm{O}_{2}$ levando à consequente formação de precipitados no meio.

Na Tabela 2, é possível observar que a porcentagem de redução de cor ICUMSA ocorreu em todos os tratamentos, quando relacionados à amostra bruta, exceto no Tratamento $16\left(\mathrm{pH} 5,50{ }^{\circ} \mathrm{C}\right.$ e $0 \mathrm{ppm}$ de $\mathrm{H}_{2} \mathrm{O}_{2}$ ), porque a amostra foi aquecida sem adição de agente clarificante. Segundo Fennema (1996), o aquecimento de carboidratos - em especial, sacarose e açúcares redutores - produz uma série de reações complexas, promotoras de cor. Dentre os tratamentos, a redução de cor ICUMSA ocorreu de forma mais intensa no Tratamento $15\left(\mathrm{pH} 5,83,6^{\circ} \mathrm{C}\right.$ e $500 \mathrm{ppm}$ de $\mathrm{H}_{2} \mathrm{O}_{2}$ ), que obteve redução de $70 \%$ da cor inicial e nos três pontos centrais do delineamento $\left(\mathrm{pH} 5,50{ }^{\circ} \mathrm{C}\right.$ e 500 ppm de $\mathrm{H}_{2} \mathrm{O}_{2}$ ), que reduziram, em média, 66\%. Diante disto, é possível apontar estes dois tratamentos como os mais satisfatórios do planejamento, para redução da cor ICUMSA inicial da calda.

Nas Figuras 1A e 1B, é possível observar que o melhor resultado de $\mathrm{pH}$ foi de 7,0 até o ponto extremo $(8,36)$, sugerindo que o planejamento fatorial apresentaria melhor resposta em uma gama maior de $\mathrm{pH}$ mais alcalinos.

Tabela 2. Porcentagem de redução de cor ICUMSA nos tratamentos de clarificação por peroxidação da calda de açúcar bruto a $66^{\circ}$ Brix após uma hora.

\begin{tabular}{|cccccc|}
\hline Tratamento & $\mathbf{p H}$ & Temperatura $\left({ }^{\circ} \mathbf{C}\right)$ & $\mathbf{H}_{\mathbf{2}} \mathbf{O}_{\mathbf{2}}(\mathbf{p p m})$ & Turbidez $(\mathbf{N T U})$ & ${ }^{2} \mathbf{I R C} \mathbf{( \% )}$ \\
\hline $\mathbf{0}$ & ${ }^{1}, 56$ & Ambiente & 0 & $17 \pm 0,06$ & - \\
$\mathbf{1}$ & 3 & 30 & 202,4 & $102 \pm 1,53$ & 15 \\
$\mathbf{2}$ & 7 & 30 & 202,4 & $111 \pm 4,36$ & 18 \\
$\mathbf{3}$ & 3 & 70 & 202,4 & $61 \pm 3,21$ & 44 \\
$\mathbf{4}$ & 7 & 70 & 202,4 & $87 \pm 25,5$ & 53 \\
$\mathbf{5}$ & 3 & 30 & 797,6 & $108 \pm 2,08$ & 36 \\
$\mathbf{6}$ & 7 & 30 & 797,6 & $128 \pm 0,58$ & 22 \\
$\mathbf{7}$ & 3 & 70 & 797,6 & $117 \pm 2,08$ & 54 \\
$\mathbf{8}$ & 7 & 70 & 797,6 & $91 \pm 15,0$ & 59 \\
${ }^{39}$ & 5 & 50 & 500 & $138 \pm 8,54$ & 67 \\
${ }^{3} \mathbf{1 0}$ & 5 & 50 & 500 & $124 \pm 22,4$ & 65 \\
${ }^{3 \mathbf{1 1}}$ & 5 & 50 & 500 & $135 \pm 6,66$ & 66 \\
$\mathbf{1 2}$ & 1,64 & 50 & 500 & $109 \pm 9,29$ & 14 \\
$\mathbf{1 3}$ & 8,36 & 50 & 500 & $115 \pm 3,21$ & 54 \\
$\mathbf{1 4}$ & 5 & 16,4 & 500 & $118 \pm 6,03$ & 61 \\
$\mathbf{1 5}$ & 5 & 83,6 & 500 & $125 \pm 2,52$ & 70 \\
$\mathbf{1 6}$ & 5 & 50 & 0 & $29 \pm 3,46$ & -7 \\
$\mathbf{1 7}$ & 5 & 50 & 1000 & $142 \pm 2,65$ & 51 \\
\hline
\end{tabular}

VValor referente ao $\mathrm{pH}$ inicial da calda de açúcar bruto a $66^{\circ}$ Brix; 2 2 ndice de redução de cor ICUMSA nas caldas de açúcar bruto a $66^{\circ}$ Brix após $1 \mathrm{~h}$; 3Ponto central do delineamento.

A

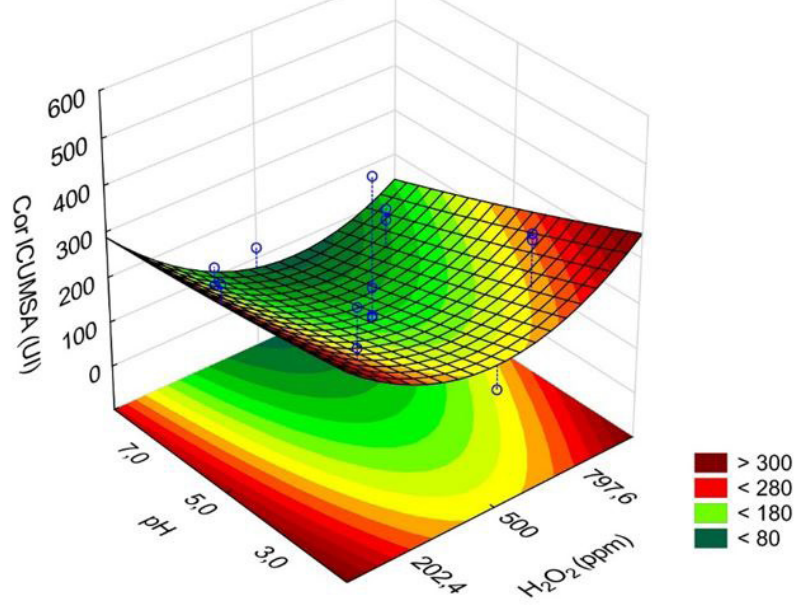

Figura 1. Superfície de resposta de cor ICUMSA para as interações das variáveis $(A) \mathrm{pH} / \mathrm{H}_{2} \mathrm{O}_{2}$ e $(\mathrm{B})$ temperatura/pH, $\mathrm{para}$ os tratamentos de clarificação da calda de açúcar bruto por peróxido de hidrogênio. 
Em estudo, Mane et al. (1992) apontaram que o uso de $\mathrm{H}_{2} \mathrm{O}_{2}$ para a redução de cor ICUMSA se mostra eficiente para teores de $\mathrm{pH}$ neutros ou alcalinos, como no caso de caldos caleados, que apresentam pH entre 7,0 e 7,2.

Na Figura 2, é possível observar pontos de relevantes reduções de cor entre a interação das variáveis $\mathrm{H}_{2} \mathrm{O}_{2}$ e temperatura, elucidando que os dois fatores propostos no modelo estatístico foram eficientes, formando uma superfície côncava com ponto ótimo entre as interações de $50{ }^{\circ} \mathrm{C}$ a $70{ }^{\circ} \mathrm{C}$ com 500 ppm a 797,6 ppm de $\mathrm{H}_{2} \mathrm{O}_{2}$. Diante desta dinâmica, foi observado que os pontos com temperatura de $50{ }^{\circ} \mathrm{C}$ e 500 ppm de $\mathrm{H}_{2} \mathrm{O}_{2}$ foram os mais representativos quanto à redução de cor ICUMSA. Em estudos, Mbanjwa et al. (2010) testaram doses de $\mathrm{H}_{2} \mathrm{O}_{2}$ de 0 a 330 ppm e, dentre estas, também observaram melhores resultados de redução de cor ICUMSA em concentração acima de 200 ppm de $\mathrm{H}_{2} \mathrm{O}_{2}$, sendo o seu melhor resultado observado com 330 ppm, em que a cor reduziu $51 \%$ em relação ao valor inicialmente medido.

O fator mais significativo (Figura 3) foi a temperatura (Quadrática; Q), seguida por $\mathrm{H}_{2} \mathrm{O}_{2}$ (Quadrática) e os demais fatores apresentam porcentagem decrescente de significância. Entretanto, observou-se que, diferentemente do termo linear da variável pH, o termo quadrático, que é representado pelos pontos extremos de $\mathrm{pH}$, não ultrapassou a linha de significância. Neste caso, é possível dizer que, entre os fatores e as interações utilizadas, o pH quadrático foi o menos significativo entre eles. Reforça-se, assim, a ideia defendida anteriormente de que os intervalos de $\mathrm{pH}$ não foram tão expressivos no planejamento fatorial estudado.

Estatisticamente, o delineamento apresentou coeficiente de variação $R=0,83$ de significância, mostrando que o tratamento explica $83 \%$ da variação dos dados experimentais. A comparação do $F_{\text {tab }}=3,29$ com o $F_{\text {cal }}=6,25$ no nível de $5 \%$ de probabilidade foi satisfatória, mostrando que o fatorial foi significativo. A falta de ajuste do modelo, quando comparado o $F_{\text {tab }}=19,35$ com o $F_{\text {cal }}=355,86$ no nível $5 \%$, também foi significativa, como é possível observar na Tabela 3. Todavia, o ideal seria um modelo com falta de ajuste não significativo.

O erro puro obtido no experimento foi de ordem extremamente baixa (Tabela 3). O teste de significância para a falta de ajuste não deve ser considerado relevante, caso os quadrados médios do erro puro sejam muito baixos (BOX; DRAPER, 1987). O modelo é válido diante do resultado obtido pelo teste $F$, mas não deve ser considerado para fins preditivos, pois, embora uma regressão seja significativa através do teste $F$, o modelo pode não ser preditivo, ou seja, este pode não servir para realizar previsões a partir do modelo gerado; então, para que uma regressão seja considerada preditiva, o valor de F calculado deve ser, no mínimo, quatro vezes o valor do F tabelado (BARROS et al., 2001).

Na Figura 4, é possível observar que a degradação da sacarose foi maior nos pontos com teores de $\mathrm{pH}$ mais
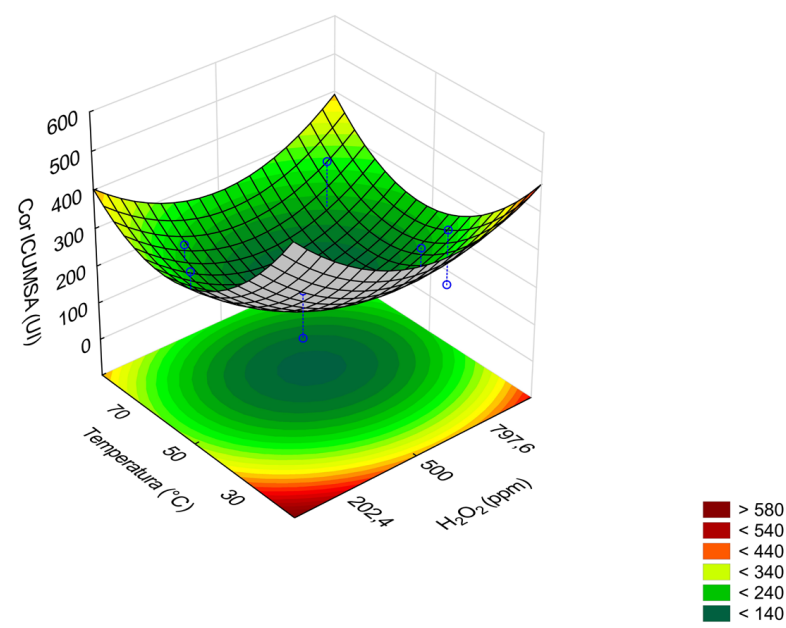

Figura 2. Superfície de resposta de cor ICUMSA para as interações das variáveis $\mathrm{H}_{2} \mathrm{O}_{2}$ /temperatura para os tratamentos de clarificação da calda de açúcar bruto por peróxido de hidrogênio.

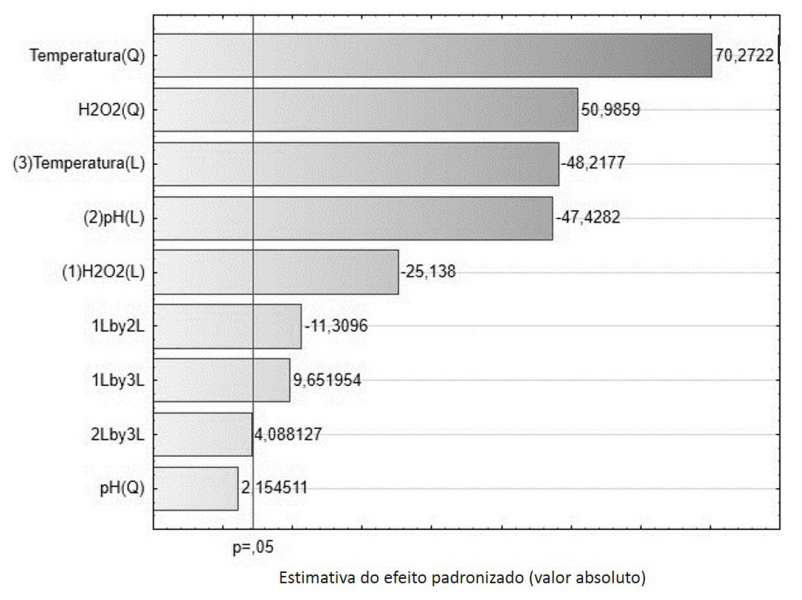

Figura 3. Diagrama de Pareto para um planejamento fatorial de cor ICUMSA envolvendo as variáveis $\mathrm{H}_{2} \mathrm{O}_{2}, \mathrm{pH}$ e temperatura, e suas interações.

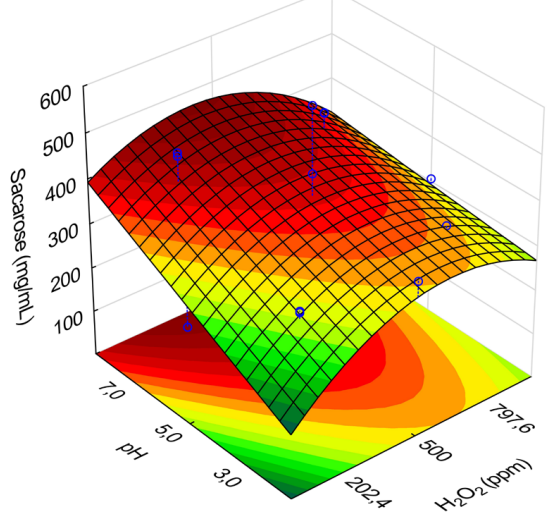

Figura 4. Superfície de resposta com interação das variáveis $\mathrm{pH} / \mathrm{H}_{2} \mathrm{O}_{2}$ para avaliar a degradação de sacarose nos tratamentos de redução de cor em calda de açúcar bruto a $66^{\circ}$ Brix, por ação do peróxido de hidrogênio. 


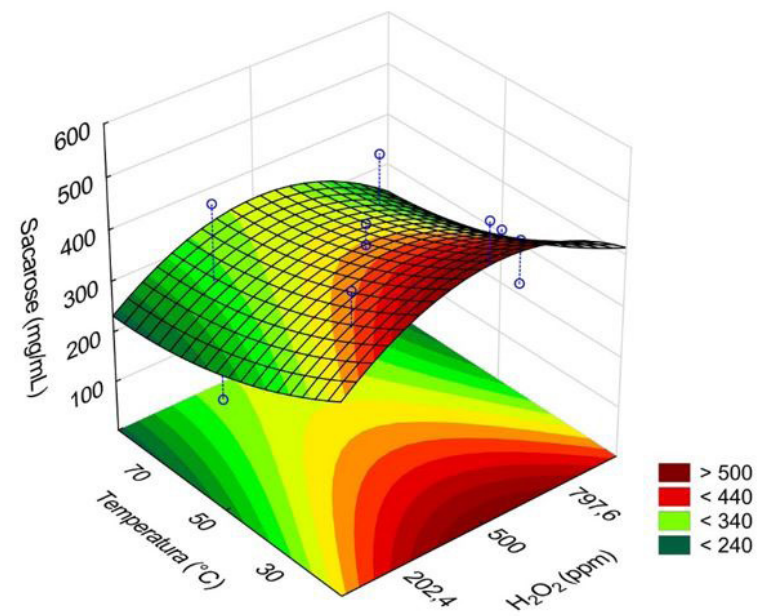

A

Figura 5. Superfície de resposta com interação das variáveis (A) temperatura/ $\mathrm{H}_{2} \mathrm{O}_{2}$ e (B) temperatura/pH para avaliar a degradação de sacarose nos tratamentos de redução de cor em calda de açúcar bruto a $66^{\circ}$ Brix, por ação do peróxido de hidrogênio.

ácidos (abaixo de 5,0), em conjunto com a concentração de $\mathrm{H}_{2} \mathrm{O}_{2}(202,4$ ppm). O fato se explica, uma vez que, em todos os tratamentos, ao final da reação, o pH da solução ficou mais ácido, em média um ponto abaixo do determinado inicialmente, o que, ao longo da reação, contribuía para a redução do potencial de degradação da sacarose dos ensaios com pH mais básicos, deixando-os mais próximos da neutralidade. Segundo Aida et al. (2007), há fortes relações entre condições de aquecimento e formação de ácidos orgânicos na solução.

Em estudo, Woo et al. (2009) também observaram que uma dada temperatura de aquecimento em tempo prolongado resultou em valores de $\mathrm{pH}$ mais baixos. A diminuição do pH com o aquecimento pode ser atribuída ao aumento da produção de ácidos orgânicos, tal como o ácido fórmico, o ácido lático e o ácido levulínico.

As Figuras 5A e 5B mostram que a temperatura, independentemente da interação, foi um fator crítico na degradação da sacarose, sendo possível observar que, em temperaturas acima de $30{ }^{\circ} \mathrm{C}$, a sacarose foi degradada em praticamente todos os pontos e interações $\left(\mathrm{pH} \mathrm{e} \mathrm{H}_{2} \mathrm{O}_{2}\right)$ estudados, com uma maior ênfase entre as interações com teores de pH abaixo de 5,0. Panpae et al. (2008) analisaram as perdas de sacarose na indústria do açúcar pela ação do pH e da temperatura, e observaram que, quando a sacarose era submetida ao aquecimento, os teores de açúcares redutores aumentavam, enquanto os de sacarose reduziam, ou seja, a temperatura age como catalisador quando o assunto é degradação de sacarose.

No diagrama de Pareto, para a degradação de sacarose (Figura 6), foi possível observar que, entre os fatores e interações estudados, apenas três foram significativos, sendo estes a interação 1Lby2 $\mathrm{L}\left(\mathrm{H}_{2} \mathrm{O}_{2}\right.$ linear interagindo com $\mathrm{pH}$ linear), $\mathrm{H}_{2} \mathrm{O}_{2}(\mathrm{~L})$ e a temperatura $(\mathrm{Q})$.

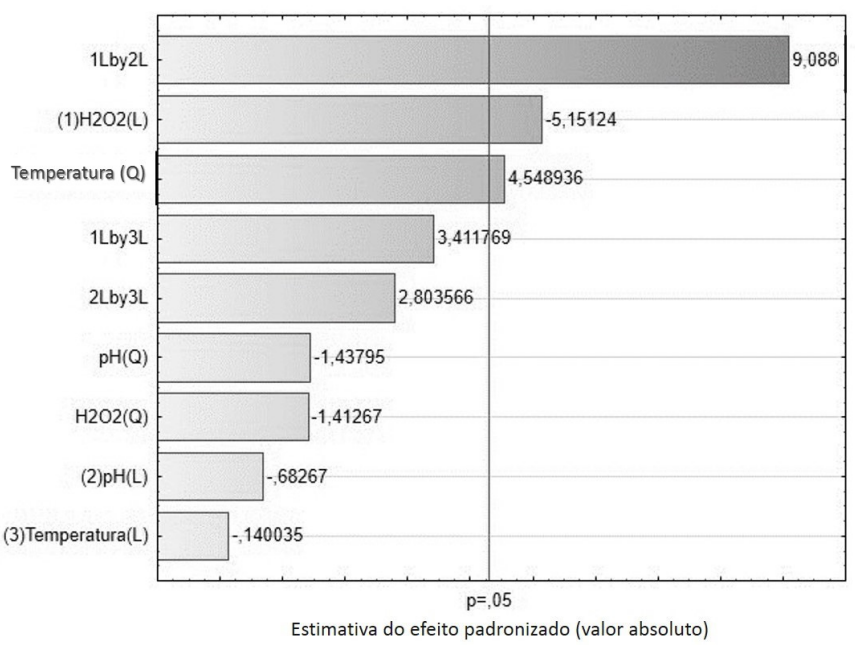

Figura 6. Diagrama de Pareto para um planejamento fatorial de degradação de sacarose envolvendo as variáveis $\mathrm{H}_{2} \mathrm{O}_{2}, \mathrm{pH}$ e temperatura, e suas interações.

Todos os outros fatores estudados não mostraram significativa importância no planejamento. Entretanto, considerando-se que a degradação de sacarose é uma condição indesejável ao tratamento, a alta incidência de fatores e interações não significativas se apresenta como um bom resultado ao fatorial, salientando que os fatores utilizados no fatorial não foram tão nocivos e significativos a ponto de inviabilizarem os tratamentos.

Estatisticamente, o tratamento fatorial para concentração de sacarose não foi significativo (Tabela 4), uma vez que a comparação do $F_{\text {tab }}=3,29$ com o $F_{\text {cal }}=1,26$, no nível de $5 \%$ de probabilidade, foi insatisfatória, como também se pode ver na Tabela 4. O modelo ainda apresentou $R=0,62$ de significância, mostrando que o tratamento consegue explicar $62 \%$ da variação dos dados experimentais. 
Redução de cor ICUMSA em soluções concentradas de açúcar bruto por processo oxidativo com peróxido de hidrogênio Mandro, J. L. et al.

Tabela 3. Análise da variância (ANOVA) do modelo ajustado $(p \leq 0,05)$, obtida a partir do coeficiente de regressão da resposta de cor ICUMSA.

\begin{tabular}{lccccc} 
& SS & DF & MS & F calculado & P \\
Regressão & 0,72 & 7 & 0,1 & 6,25 & 0,01 \\
Residual & 0,15 & 9 & 0,02 & & \\
Falta de ajuste & 0,15 & 7 & 0,02 & 35,86 & 0 \\
Puro erro & 0 & 2 & 0 & & \\
Total & 0,86 & 16 & 0,05 & & \\
\hline
\end{tabular}

Tabela 4. Análise da variância (ANOVA) do modelo ajustado $(p \leq 0,05)$, obtida a partir do coeficiente de regressão da resposta de degradação de sacarose.

\begin{tabular}{lccccc} 
& SS & Df & MS & F calculado & P \\
Regressão & 0,28 & 9 & 0,03 & 1,26 & 0,39 \\
Resíduo & 0,17 & 7 & 0,02 & & \\
Falta de ajuste & 0,16 & 5 & 0,03 & & 0,15 \\
Erro puro & 0,01 & 2 & 0,01 & & \\
Total & 0,44 & 16 & 0,03 & & \\
\hline
\end{tabular}

\section{Conclusões}

O peróxido de hidrogênio foi eficiente na redução de cor ICUMSA da calda de açúcar VHP, principalmente para os pontos centrais do delineamento. A precipitação dos compostos ocorreu em consequência das dosagens de $\mathrm{H}_{2} \mathrm{O}_{2}$, pois se verificou que, quanto maior foi a aplicação do agente clarificante, maior foi o aumento de partículas no meio. A degradação da sacarose ocorreu em todos os tratamentos, porém as condições necessárias para obtenção de menor cor ICUMSA foram as menos relacionadas à degradação da sacarose.

\section{Agradecimentos}

Os autores agradecem à FAPESP (processos $n^{\circ}$ 2009/54635-1 e 2014/03292-5) e ao CNPq (processo $\left.n^{\circ} 310367 / 2013-1\right)$ pelo apoio financeiro durante o desenvolvimento dos projetos de pesquisa.

\section{Referências}

AIDA, T. M.; TAJIMA, K.; WATANABE, M.; SAITO, Y.; KURODA, K.; NONAKA, T.; HATTORI, H.; SMITH, R. L.; ARAI, K. Reactions of $\mathrm{D}$ - fructose in water at temperatures up to $400{ }^{\circ} \mathrm{C}$ and pressures up to $100 \mathrm{MPa}$. The Journal of Supercritical Fluids, Cincinnati, v. 42, n. 1, p. 110-119, 2007. http://doi.org/10.1016/j. supflu.2006.12.017

ASSOCIAÇÃO BRASILEIRA DE NORMAS TÉCNICAS - ABNT. NBR 9757: açúcar: determinação de turbidez. Rio de Janeiro, $1987.5 \mathrm{p}$.

BACCAR, R.; BOUZID, J.; FEKI, M.; MONTIEL, A. Preparation of activated carbon from Tunisian olive-waste cakes and its application for adsorption of heavy metal ions. Journal of Hazardous Materials, Amsterdam, v. 162, n. 2-3, p. 1522-1529, 2009. PMid:18653277. http://dx.doi.org/10.1016/j.jhazmat.2008.06.041.
BARROS, N. B.; SCARMíNIO, I. S.; BRUNS, R. E. Como fazer experimentos: pesquisas e desenvolvimento na ciência e na indústria. Campinas: Editora da UNICAMP, 2001. 401 p.

BOURZUTSCHKY, H. C. C. Color formation and removal: options for the sugar and sugar refining industries: a review. Zuckerindustrie, Berlin, v. 130, n. 7, p. 545-553, 2005.

BOX, G. E. P.; DRAPER, N. R. Empirical model-building and response surfaces. 3. ed. New York: John Wiley, 1987. 669 p.

CAHILL, A. E.; BURKHART, L. E. Continuous precipitation of uranium with hydrogen peroxide. Metallurgical Transactions, Ames, v. 21, n. 5, p. 819-826, 1990. http://dx.doi.org/10.1007/ BF02657806.

CREMA, L. C. Clarificação por flotação com ar dissolvido (FAD) da calda de açúcar cristal para produção de açúcar refinado. 2012. 128 f. Dissertação (Mestrado em Engenharia e Ciência de Alimentos)-Universidade Estadual Paulista, São José do Rio Preto, 2012.

DJOGIC, R.; CUCUCLIC, V.; BRANICA, M. Precipitation of uranium (VI) peroxide (UO4) in sodium perchlorate solution. Croatica Chemica Acta, Croatia, v. 78, p. 575-580, 2005.

FENNEMA, O. R. Food chemistry. 3rd ed. New York: Marcel Dekker, Inc., 1996. 1262 p.

INTERNATIONAL COMMISSION FOR UNIFORM METHODS OF SUGAR ANALYSIS - ICUMSA. Determination of the solution colour of raw sugar, brown sugar and coloured syrups at pH 7.0 - Official. Berlin: ICUMSA, 2011. (ICUMSA Method GS 1/3-7 - Raw Sugar).

MACHADO, S. S. Tecnologia da fabricação do açúcar. Inhumas: Instituto Federal de Educação, Ciência e Tecnologia, 2012. Rede E-tec Brasil. 
Redução de cor ICUMSA em soluções concentradas de açúcar bruto por processo oxidativo com peróxido de hidrogênio Mandro, J. L. et al.

MANE, J. D.; PHADNIS, S. P.; JADHAV, S. J. Effects of hydrogen peroxide on cane juice constituents. International Sugar Journal, London, v. 94, n. 1128, p. 322-324, 1992.

MARQUEZ, L. D. S. Produção de açúcar invertido pelo uso de invertase imobilizada em resinas. 2007. 107 f. Dissertação (Mestrado em Engenharia de Química)-Universidade Federal de Uberlândia, Uberlândia, 2007.

MBANJWA, C. F.; DEPPA, N.; PILLAY, K. Evaluation of hydrogen peroxide at huletts refinery: preliminary results. International Sugar Journal, London, v. 113, n. 1349, p. 343-347, 2010.

NIKONOV, M. V.; TANANAEV, I. G.; MYASOEDOV, B. F. Effect of hydrogen peroxide on precipitation of $\mathrm{Pu}(\mathrm{IV})$ in alkaline solutions. Radiochemistry, Switzerland, v. 52, n. 1, p. 27-30, 2010. http:// dx.doi.org/10.1134/S1066362210010066.

PANPAE, K.; JATURONRUSMEE, W.; MINGVANISH, W.; NUNTIWATTANAWONG, C.; CHUNWISET, S.; SANTUDROB, K.; TRIPHANPITAK, S. Minimization of sucrose losses in sugar industry by $\mathrm{pH}$ and temperature optimization. The Malaysian Journal of Analytical Sciences, Bangi, v. 12, n. 3, p. 513-519, 2008.

RODRIGUES, M. V. N. Otimização da produção de xarope de açúcar invertido através do uso de resinas de troca-iônica.
1998. 107 f. Dissertação (Mestrado em Engenharia de Alimentos)Universidade Estadual de Campinas, Campinas, 1998.

SARTORI, J. A. S.; GALAVERNA, R.; EBERLIN, M. N.; CORREA, N. T.; MANDRO, J. L.; AGUIAR, C. L. Elucidation of color reduction involving precipitation of non-sugars in sugarcane (Saccharum sp.) juice by fourier-transform ion cyclotron resonance mass spectrometry. Journal of Food Processing and Preservation, Malden, v. 39, n. 6, p. 1826-1831, 2015a. http://dx.doi.org/10.1111/ jfpp. 12417.

SARTORI, J. A. S.; CORREA, N. T. C.; AGUIAR, C. L. Clarificação de caldo de cana-de-açúcar por peróxido de hidrogênio: efeito da presença de dextrana. Brazilian Journal of Food Technology, Campinas, v. 18, n. 8, p. 299-306, 2015b. http:// dx.doi.org/10.1590/1981-6723.4215.

SILVA, S. W.; SARTORI, J. A. S.; AGUIAR, C. L. Combination effect of ozone and heat treatment for the color reduction in sugarcane juice. Chemical and Process Engineering Research, New York, v. 35, p. 73-83, 2015.

WOO, K. S.; HWANG, I. G.; LEE, Y. R.; LEE, J.; JEONG, H. $S$. Characteristics of sucrose thermal degradation with high temperature and high pressure treatment. Food Science and Biotechnology, Seoul, v. 18, n. 3, p. 717-723, 2009. 